\title{
Down syndrome and aberrant right subclavian artery
}

\author{
Marcus T. R. Roofthooft • Hester van Meer • \\ Wim G. Rietman • Tjark Ebels • Rolf M. F. Berger
}

Received: 20 July 2007 / Accepted: 6 November 2007 / Published online: 3 January 2008

(C) The Author(s) 2007

\begin{abstract}
Down syndrome (DS) may be associated with various organ system disorders. Feeding problems are frequent in children with DS and may be caused by associated defects, including congenital heart defects, gastrointestinal defects, or endocrine disorders. In the absence of these associated conditions, feeding problems are often attributed to general hypotonia. However, an aberrant right subclavian artery (ARSA), a rare vascular anomaly and an unusual cause of problems with the passage of solid food through the esophagus, has recently been suggested to occur more frequently in patients with DS. This knowledge is of importance when evaluating feeding difficulties in patients with DS. Additional investigation for identifying an ARSA may be indicated in selected patients. Diagnostic techniques, such as transthoracic echocardiography, barium contrast esophagram, angiography, or computed tomography-angiography (CT) can be used in a diagnostic flow chart. The presence of ARSA
\end{abstract}

M. T. R. Roofthooft $(\bowtie) \cdot$ H. van Meer $\cdot$ R. M. F. Berger Department of Paediatric Cardiology, Beatrix Children's Hospital, University Medical Centre Groningen, University of Groningen, Hanzeplein 1,

9700 Groningen, The Netherlands

e-mail: M.T.R.Roofthooft@BKK.UMCG.nl

W. G. Rietman

Department of Anaesthesiology,

University Medical Centre Groningen,

University of Groningen,

Groningen, The Netherlands

T. Ebels

Department of Cardiothoracic Surgery,

University Medical Centre Groningen,

University of Groningen,

Groningen, The Netherlands is not synonymous to the cause of feeding problems in patients with DS and corrective surgery of this vascular anomaly should be restricted to selected cases.

Keywords Down syndrome - Feeding problems . Aberrant right subclavian artery (ARSA) .

Imaging techniques · Surgery
Abbreviations
ARSA Aberrant right subclavian artery
CT Computed tomography
DS Down syndrome
c-AVSD Complete atrioventricular defect
MR Magnetic resonance
PPHN Persistent pulmonary hypertension of the newborn
TEE Trans esophageal echocardiography

\section{Introduction}

Down syndrome (DS), trisomy 21 , is an autosomal disorder with an incidence of 1 to 1.7 per 1,000 births and a variable physical expression $[6,9,10]$. Feeding problems in infants and young children with DS are frequent and may lead to failure to thrive. The feeding problems may be caused by underlying conditions frequently associated with DS. These include congenital heart defects (present in $40-60 \%$ of DS patients), gastrointestinal disorders such as duodenal web, gastro-esophageal reflux, endocrine disorders, or impaired oral motor function. In the absence of underlying disease conditions, feeding difficulties in DS are often attributed to the general hypotonia and related impaired oral motor function. Detailed history taking, emphasising the age of presenting symptoms as well as the type of feeding, and 


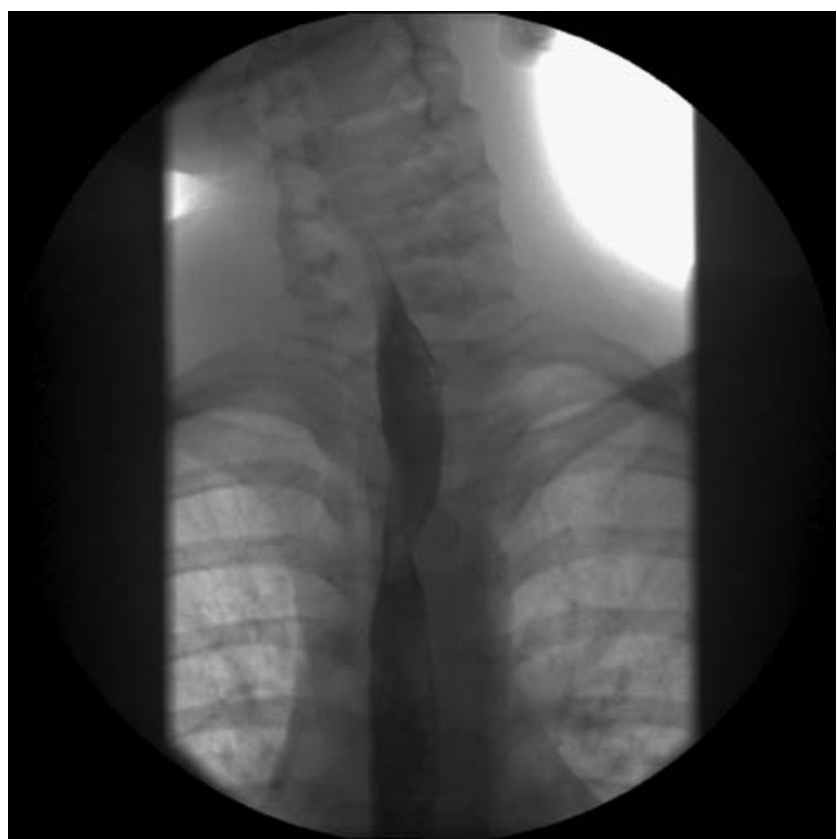

Fig. 1 Barium contrast examination of the esophagus

observation of the feeding pattern may provide clues to the cause of the feeding difficulties. For example, progressive dyspnea and sweating during feeding may be a sign of impaired exercise capacity due to a congenital cardiac defect, whereas vomiting, early or late after ingestion, or position-related complaints may suggest a gastrointestinal tract disorder. In the presence of a cardiac defect, it can be difficult to differentiate between the possible causes. The sequence and range of required diagnostic procedures will be directed by the clinical symptoms in these patients.

An aberrant right subclavian artery (ARSA) is a rare vascular anomaly that is believed to induce feeding and swallowing difficulties in $20 \%$ of the patients, caused by dorsal compression of the esophagus by the anomalous artery. Recently it has been suggested that the prenatal occurrence of this vascular anomaly is substantially increased in DS where it can be found in up to $19-36 \%$ of cases [4].

In the evaluation of feeding problems in patients with DS this association should be kept in mind, but also the comprehension that the presence of ARSA will often not be the cause of feeding difficulties. This is illustrated in the following patient histories, representing our institution's experience with this association in a 2 year period (2005-2006) in which ARSA was diagnosed in three children with DS.

\section{Patients}

The first patient is a girl with DS and a complete atrioventricular septal defect (c-AVSD). She suffered from feeding difficulties presenting shortly after birth. Despite adequate surgical correction of the c-AVSD, feeding difficulties persisted, which were then attributed to mild hypotonia. She was tube fed for nearly 2 years. Introduction of solid food was accompanied by difficulties in swallowing and progressive vomiting, eventually leading to reluctance of solid food. The family history showed no presence of atopy or feeding problems. She was treated and guided by a feeding team. However, a barium contrast examination of the esophagus made at the age of 6 years revealed an impression of the esophagus which suggested an ARSA (Fig. 1).

Transthoracic echocardiography could not identify an aberrant course of the aortic branch vessels due to limited proper views. Multi-slice computed tomography (CT) confirmed the diagnosis ARSA (Fig. 2). At the age of 7 years she underwent surgical correction, performed through a left thoracotomy in the fourth intercostal space. An ARSA (lusorian artery) originated from the aortic isthmus, distally from the left subclavian artery, and compressed the esophagus dorsally. The aberrant vessel was divided and oversewn at both the aortic side as well as the lusorian artery side, thus freeing the esophagus from its dorsal compression. During follow-up, dysphagial complaints gradually relieved over a period of 3 months. She currently has a normal feeding pattern.

The second patient is a boy with DS and a history of persistent pulmonary hypertension of the newborn (PPHN) and a c-AVSD. Due to progressive tachypnea, nasogastric tube feeding was required. After recovery from PPHN, feeding problems persisted which consisted of swallowing difficulties and frequent vomiting. Surgical correction of the c-AVSD was performed at the age of 4 months. During this procedure it was noticed that introduction of the transesophageal echocardiography (TEE) probe caused a

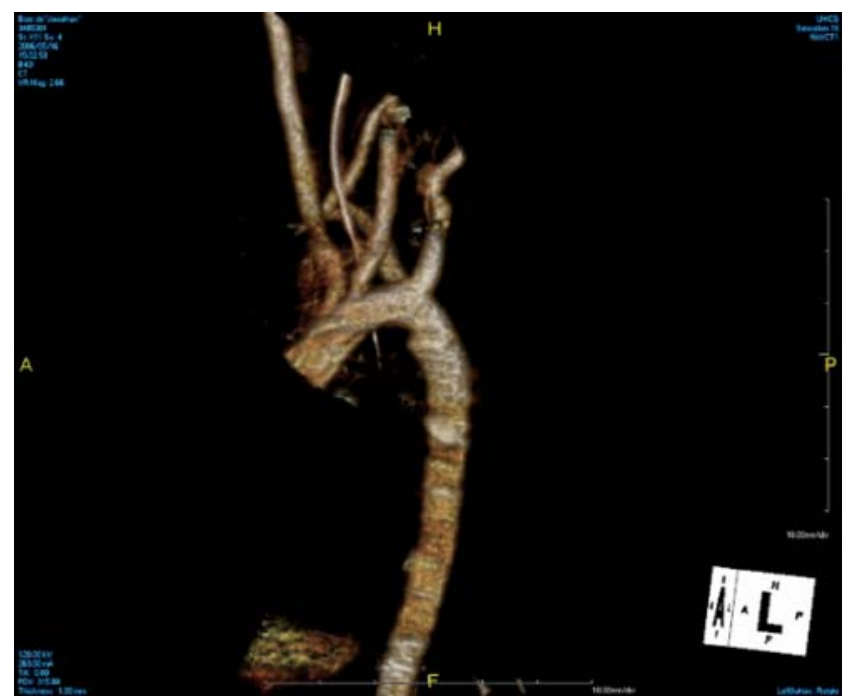

Fig. 2 CT-3D volume rendering (Aquarius Net Viewer V1.6.0.8, TeraRecon, San Mateo, CA, USA). An aberrant right subclavian artery forms the fourth side-branch 
drop in arterial blood pressure (measured invasively in the right radial artery) without a change in heart frequency. This is a feature suggestive to the presence of a compressing vascular structure against the esophagus, which, however, was not recognised at that time [7]. Because of the combination of persistent feeding problems (impaired swallowing and frequent vomiting despite adequate oral motor function) and the lack of a satisfactory cause (strengthened by the TEE feature), a CT-angiography was performed at the age of 1 year that revealed an ARSA. The patient subsequently underwent surgical correction of the ARSA. Nevertheless, after 1 year of follow-up, the feeding problems had not improved and the patient is still fed through a gastrostomy.

In the third patient, a girl with DS and c-AVSD complicated by symmetrical left ventricular hypertrophia of unknown origin, an ARSA was accidentally discovered during delayed primary surgical correction at the age of 11 months. The aberrant vessel was divided and oversewn at both the aortic side as well at the lusorian artery side. In this patient with severe hypotonia and retardation, feeding problems did not relieve in the following year. She is now fed through a gastrostomy.

\section{Discussion}

Feeding problems are frequent in infants and young children with DS and can be caused by a variety of etiologies. Therefore, in such patients an individualized diagnostic work-up has to be performed, guided by the pattern of the feeding difficulties, clinical symptoms, and knowledge of associated conditions. Congenital heart defects are present postnatally in $40-60 \%$ of patients with DS with c-AVSD being the most common defect [8]. These defects, associated with important left-to-right shunting, often form a prominent factor in feeding difficulties in the first months of life. Because of this high incidence, early cardiologic screening, including echocardiography, is recommended in order to detect and correct CHD early in life. In the presence of a congenital heart defect it may be difficult to assess the possible presence of other factors affecting feeding pattern. However, after adequate surgical correction with restoration of hemodynamic balance, CHD should no longer cause feeding difficulties. In cases where no associated condition explaining the complaints is detected, feeding problems are often attributed to general hypotonia or oral motor dysfunction, frequently present in young children with DS. Our first patient, however, illustrates that an easily treatable structural anomaly may remain undetected for many years as a cause for feeding difficulties.

The term arteria lusoria, literally meaning "playful artery", was introduced by Bayford in 1794 [2]. This lusorian artery is a relatively rare anomaly in which the right subclavian artery arises from the aortic arch distal of the left subclavian artery and crosses the midline behind the esophagus (Fig. 3). The ARSA may cause symptoms such as dysphagia associated with solid food; however, this is only seen in a minority of cases $(10-20 \%)$ [1]. Consequently, in young formula-fed infants the presence of this vascular anomaly is highly unlikely to be the cause of feeding difficulties, as illustrated by our second patient. Only after the introduction of solid food and a history compatible with obstructed passage of solid food should the presence of ARSA be considered as causative for feeding problems.

The incidence of ARSA in DS has been reported in up to $16-39 \%$ of cases by Chaoui et al. [4]. It should be noted that this report refers to prenatal echocardiographic findings in 14 patients. Based on these data an association between DS and ARSA is suggested. Recognition of this association is of importance when evaluating feeding or swallowing difficulties in DS patients. However, these findings have to be confirmed and the exact postnatal incidence of ARSA in DS remains to be established.

Another aspect of this high prenatal incidence of ARSA in DS may be its use in prenatal diagnosis of DS. Presently, the gold standard in prenatal screening consists of chromosomal karyotyping of fetal cells, obtained by either chorionic villus sampling (9-11 weeks of gestation) or amniocentesis (16-18 weeks of gestation). Both methods are accompanied by the risk of miscarriage [5]. The triple

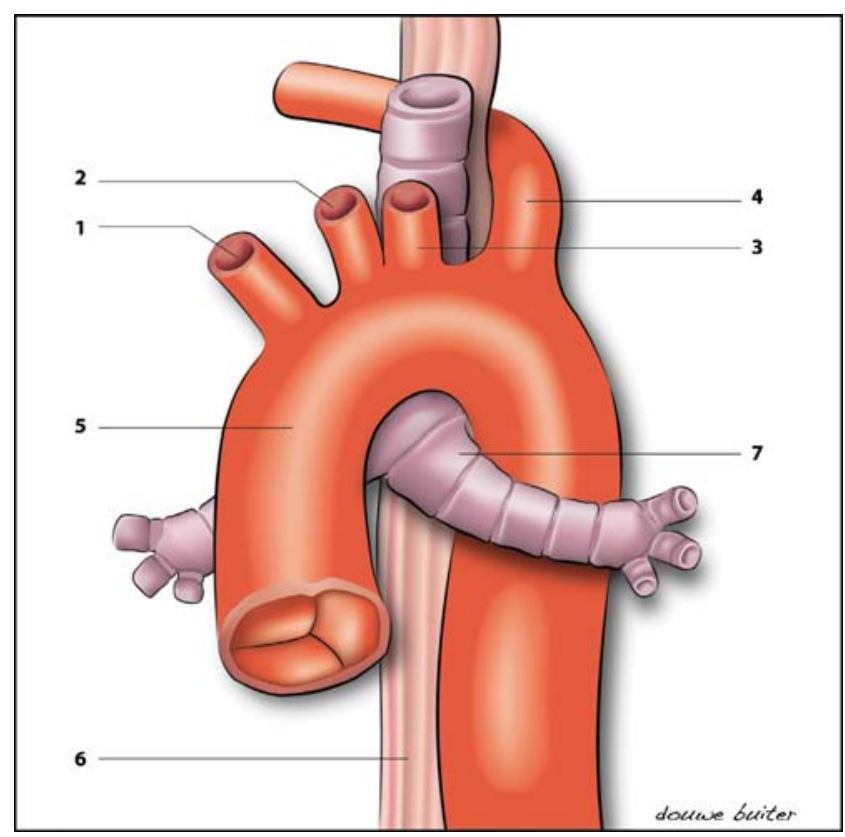

Fig. 3 Anatomy of an aberrant right subclavian artery. Right common carotid artery (1), left common carotid artery (2), left subclavian artery (3), aberrant right subclavian artery (4), ascending aorta (5), esophagus (6), and left bronchus (7) 
screen test (maternal serum levels of $\alpha$-fetoprotein, unconjugated estriol, and $\beta$-human-chorionic gonadotropin) in combination with ultrasound investigation of nuchal translucency performed in the second trimester, as well as short femurs, cardiac anomalies, and duodenal atresia, can strengthen the suspicion of DS. Chaoui et al. suggest that the in utero identification of an ARSA may be an additional new ultrasound marker in second- and third-trimester fetuses with DS that might expand the predictability of DS. Although prenatal visualisation of aortic branch vessels may be difficult, Chaoui et al. were able to visualise normal and abnormal branching of the right subclavian artery between 18 and 33 weeks of gestation in all 14 patients with earlier detected DS [4].

In cases of suspected postnatal ARSA, the diagnostic approach has changed over the years, from the 1940s with barium contrast esophagrams, through the 1960-1970s with angiography, to the 1990s until present with CT and magnetic resonance (MR) imaging [3]. Invasive angiography was used as the gold standard for decades; however, less invasive imaging techniques, such as $\mathrm{CT}$ and MR imaging, show equal efficiency. In our practice, when an ARSA is suspected, we start with a transthoracic echocardiography and, when negative, a barium contrast esophagram. This is because transthoracic echocardiography is noninvasive, easily accessible, and can exclude or define associated cardiac anomalies but has a low sensitivity in detecting ARSA; in contrast, the barium contrast esophagram provides a high sensitivity in this regard [1]. In cases of identification of ARSA (or persisting suspicion), we subsequently perform CT-angiography of the aorta to confirm an ARSA and to elucidate the involved anatomy. Because of the need for breath-holding, magnetic resonance imaging is less suitable for small children.

The patient histories presented here provide a two-sided message. On the one hand, ARSA is a vascular anomaly associated with DS and may be less rare in patients with DS than in the normal population. This knowledge is of importance when evaluating feeding difficulties in DS patients. On the other hand, the presence of ARSA is not synonymous with the cause of feeding difficulties. Only a minority of patients with ARSA (10-20\%) present with feeding problems, referred to as "dysphagia lusoria". These problems classically consist of restricted passage of solid food through the esophagus. Therefore, in the author's opinion, the presence of ARSA only justifies surgical intervention in those patients in whom the classical pattern of solid feeding passage difficulties "dysphagia lusoria" is present.

Obviously, these are not formula-fed young infants. In young patients with ARSA who undergo a surgical correction of concomitant congenital heart defect, ligation, or reimplantation of the ARSA can be considered.

Acknowledgements The authors would like to thank Douwe Buiter for his illustration (Fig. 3).

Open Access This article is distributed under the terms of the Creative Commons Attribution Noncommercial License which permits any noncommercial use, distribution, and reproduction in any medium, provided the original author(s) and source are credited.

\section{References}

1. Bakker D, Berger R, Witsenburg M, Bogers A (1999) Vascular rings: a rare cause of common respiratory symptoms. Acta Paediatr 88:947-952

2. Bayford D (1794) An account of a singular case of obstructed deglutition. Mem Med Soc London 2:275-280

3. Berdon W (2000) Rings, slings, and other things: vascular compression of the infant trachea updated from the mid century to the millenium - the legacy of Robert E. Gross, MD, and Edward B.D. Neuhauser, MD. Radiology 216:624-632

4. Chaoui R, Heling K, Sarioglu N, Schwabe M, Dankof A, Bollmann R (2005) Aberrant right subclavian artery as e new cardiac sign in second- and third-trimester fetuses with Down syndrome. Am J Obstet Gynaecol 192:257-263

5. Gramellini D, Fieni S, Casilla G, Raboni S, Nardelli G (2007) Mid-trimester amniocentesis and antibiotic prophylaxis. Prenat Diagn 27:956-959

6. Hahn J, Shaw G (1993) Trends in Down syndrome prevalence in California, 1983-1988. Paediat Perinatal Epidemiol 7:450-460

7. Koinig H, Schlemmer M, Keznicki F (2003) Occlusion of the right subclavian artery after insertion of a transesophageal echocardiography probe in a neonate. Peadiatr Anaesth 13: 617-619

8. Marino B (1996) Patterns of congenital heart disease and associated cardiac anomalies in children with Down syndrome. In: Marino B, Pueschel SM (eds) Heart disease in persons with Down syndrome. Brookes, Baltimore, pp 145-150

9. Saenz R (1999) Primary care of infants and young children with Down syndrome. Am Fam Physician 59:381-390

10. Stoll C, Alembik Y, Dott B, Roth M (1998) A study of Down syndrome in 238,942 consecutive births. Ann Genet 41:44-51 